\title{
Electron Emission of Graphene-Diamond Hybrid Films Using Paraffin Wax as Diamond Seeding Source
}

\author{
Deepak Varshney ${ }^{1,2^{*}}$, Chitturi Venkateswara Rao $^{3}$, Frank Mendoza ${ }^{1,2}$, Kenneth Perez ${ }^{1,2}$, \\ Maxime J.-F. Guinel ${ }^{1,2,3}$, Yasuyuki Ishikawa ${ }^{2,3}$, Brad R. Weiner ${ }^{2,3}$, Gerardo Morell ${ }^{1,2}$ \\ ${ }^{1}$ Department of Physics, University of Puerto Rico, San Juan, USA \\ ${ }^{2}$ Institute of Functional Nanomaterials, University of Puerto Rico, San Juan, USA \\ ${ }^{3}$ Department of Chemistry, University of Puerto Rico, San Juan, USA \\ Email: *deepvar20@gmail.com
}

Received June 6, 2012; revised July 3, 2012; accepted July 20, 2012

\begin{abstract}
We present a scalable, reproducible and economic process for the fabrication of diamond and diamond-graphene hybrid films using paraffin wax as a seeding source for diamond. The films were characterized using Raman spectroscopy, scanning electron microscopy (SEM), transmission electron microscopy (TEM), and electron energy loss spectroscopy (EELS). Raman spectra show the characteristic band of diamond at $1332 \mathrm{~cm}^{-1}$ and the D, G, and 2D bands of graphene at 1360,1582 and $2709 \mathrm{~cm}^{-1}$, respectively. Electron microscopy confirms the microcrystalline nature of the diamond films with crystal size in the range of $0.5 \mu \mathrm{m}$ to $1.0 \mu \mathrm{m}$, and the hybrid film consists of microcrystalline diamond attached to thin, semi-transparent graphene flakes. The graphene-diamond hybrid films exhibit a turn-on field of about 3.6 $\mathrm{V} / \mu \mathrm{m}$ with a prolonged current stability of at least $135 \mathrm{~h}$.
\end{abstract}

Keywords: Graphene; Chemical Vapor Deposition; Diamond Films; Electron Field Emission

\section{Introduction}

The exceptional properties (e.g. high thermal conductivity, high strength, and lowest compressibility) of diamond make it an ideal material for many applications, such as in cutting tools, coatings for magnetic disks, optical switches [1], electronic devices [2], spintronics devices, and quantum computational components [3] and more. Various surface pre-treatment methods have been used to enhance the nucleation density of diamond [4-8]. All these reported methods lead to surface alteration or damage (usually by the formation of nano-scale pits, scratches and defects concentration) and even contaminate the substrate. Furthermore, surface pre-treatment cannot be easily applied to substrates with complex geometries, and is often incompatible with industrial applications because of the increased cost. It is therefore important to nucleate diamond on an electrically conducting surface without the need for surface pre-treatment while maintaining high nucleation density and good adhesion.

Isolated graphene, a one-atom-thick layer of graphitic carbon, was first reported in 2004 [9], and has been dubbed as the wonder material. It opens new opportunities because of its unique electrical and mechanical properties. Its potential applications are many, including

\footnotetext{
"Corresponding author.
}

solar cells, field-emission devices and batteries [10,11]. The field emission properties of diamond [12], amorphous carbon [13], and vertically aligned multi- and single-walled carbon nanotubes [14,15] for cold cathode applications have been extensively investigated but the field emission properties of graphene films have only been reported very recently $[16,17]$. These studies on the field emission from various carbon materials focused on the importance of the field enhancement factor according to the morphological and topographical structure of the cathode surface. The excellent electrical and thermal properties of graphene and diamond respectively can be combined into one hybrid material for advanced applications.

We have recently reported the direct nucleation of diamond from kitchen wrap-polyethylene [18]. In the present work, we report a new method for diamond nucleation using paraffin wax as the seeding material. Graphenediamond hybrid films were also fabricated using the same seeding source for diamond. The method hereby described is simple, cost effective and handy. The graphene-diamond hybrid films show excellent field emission characteristics with a low turn-on field and are able to withstand for longer times compared to graphene alone. 


\section{Experimental}

For the synthesis of diamond films, paraffin wax (ca. $5 \mathrm{~g}$ ) was melted on a hot plate and a small portion of the melt was transferred onto a copper disk substrate $(14 \mathrm{~mm}$ diameter) with the help of a glass dropper and allowed to cool to room temperature. It was then introduced into the HFCVD chamber. This wax coated copper substrate was exposed to a gas mixture consisting of $0.3 \%$ methane in hydrogen with $10 \%$ methane and $99.7 \%$ of hydrogen for $4 \mathrm{~h}$, at a constant pressure of 20 Torr and a total gas flow of $100 \mathrm{sccm}$. This gaseous mixture was activated by a rhenium filament $(8 \mathrm{~cm}$ in length and $0.5 \mathrm{~mm}$ in diameter) positioned $9 \mathrm{~mm}$ above the substrate. The temperature of the substrate and the filament was kept at approximately $450^{\circ} \mathrm{C}$ and $2500^{\circ} \mathrm{C}$, respectively.

The graphene-diamond hybrid films were fabricated using a similar procedure. An amount of $0.02 \mathrm{~g}$ of presynthesized graphene powder [19] was mixed with the paraffin melt to obtain a homogeneous suspension which was then transferred onto a copper disk substrate and subjected to the same reaction conditions as those used in the fabrication of pure diamond films.

\section{Method of Characterization}

The Raman scattering spectra were obtained on a triple monochromator (ISAJ-Y Model T 64000) with approximately $1 \mathrm{~cm}^{-1}$ resolution using the $514.5 \mathrm{~nm}$ line of Ar laser. Scanning electron microscopy (SEM) images were recorded using a JEOL JSM-7500F SEM also equipped with a transmission electron detector. The samples were analyzed using a Carl Zeiss LEO 922 energy filtered transmission electron microscope (TEM) operated at 200 $\mathrm{kV}$.

The field emission I-V characteristics of the fabricated films were measured using a custom built system [20, 21].

\section{Results and Discussion}

\subsection{Raman Analysis}

The First, The paraffin wax crystallites act as nucleation sites for diamond growth in the presence of hydro-carbon radicals and atomic hydrogen in the CVD system. When the melted paraffin wax cools below its equilibrium melting temperature, its crystallization via self-nucleation becomes thermodynamically favorable and it acts as a seeding source for diamond nucleation. Also, in the present case, the use of non-carbide forming substrate is important as it provides a surface with minimal surface interactions, facilitating the formation of crystallites in the lowest energy diamond shape $[22,23]$.

The first order Raman spectrum of the wax-coated substrate is shown in the inset of Figure 1(a). It clearly shows distinctive Raman contributions at $1063 \mathrm{~cm}^{-1}$,
$1133 \mathrm{~cm}^{-1}, 1296 \mathrm{~cm}^{-1}$, and $1441 \mathrm{~cm}^{-1}$. These are attributed to $\mathrm{C}-\mathrm{C}$ (carbon-carbon) stretching and $\mathrm{CH} 2$ and $\mathrm{CH}_{3}$ deformation [24], as would be expected given the straight-chain hydrocarbon structure of wax. Figure 1(a) shows the Raman spectrum recorded from the film obtained from the CVD process of wax coated copper substrate. The intense band at $1332.6 \mathrm{~cm}^{-1}$ is characteristic of diamond [25]. The disappearance of the typical vibrational modes of paraffin wax and the appearance of band at $1332.6 \mathrm{~cm}^{-1}$ clearly indicates the growth of diamond films from paraffin wax. The fabrication of diamond film using paraffin wax as a seeding source follows the same mechanism as reported in case of kitchen wrap polyethylene [18].

The Raman spectrum of the pre-synthesized graphene is shown in inset of Figure 1(b) showing the presence of $\mathrm{D}$ band corresponding to the disorder induced in $\mathrm{sp}^{2}$ carbon [26] and a band around $1582 \mathrm{~cm}^{-1}$ corresponds to G-band [27]. The position and shape of the prominent 2D peak in the Raman spectrum can be used to clearly distinguish the number of layers in the graphene sample [28]. Broad 2D band observed at $2685 \pm 5 \mathrm{~cm}^{-1}$ in different regions indicate that the material is few layered ( 5 - 10 layers) graphene. The Raman spectrum of the graphene-diamond hybrid material in the range $1000-3000$ $\mathrm{cm}^{-1}$ is shown in Figure 1(b). The band at $1582 \mathrm{~cm}^{-1}$ corresponds to the $\mathrm{G}$ band arising from the in-plane vibrations of $\mathrm{sp}^{2}$ carbon atoms of graphene, or the doubly degenerate (TO and LO) phonon mode (E2 g symmetry) at the Brillouin zone center. The band at $1332.6 \mathrm{~cm}^{-1}$ remains present and is that of diamond $\left(\mathrm{sp}^{3} \mathrm{C}\right)$. A shoulder at $1360 \mathrm{~cm}^{-1}$ is identified as the D-band which is attributed to the disorder-induced in $\mathrm{sp}^{2}$-bonded carbon [29]. The 2D band at $2709 \mathrm{~cm}^{-1}$ originates from a two phonon double resonance Raman process and is closely related to the band structure of graphene and is used to confirm the presence of graphene [30]. The Raman spectrum in the range of $2600-2900 \mathrm{~cm}^{-1}$ is shown in the inset of Figure 1(b). It clearly depicts two bands at 2709 and $2845 \mathrm{~cm}^{-1}$ corresponding to the $2 \mathrm{D}$ and $\mathrm{S} 3$ peaks for graphene [31]. The 2D band, which is a characteristic of graphene, is used to determine the number of layers of graphene in the sample [32]. The broad and less intense 2D band seen here indicates that the hybrid material consists of few layered graphene.

\subsection{Scanning Electron Microscopy}

The morphology of the diamond and graphene-diamond hybrid films was ascertained by scanning electron microscopy (SEM). Figure 2(a) shows an overview SEM image of the diamond film composed of microcrystalline diamond. The inset of Figure 2(a) shows a diamond crystal with well-defined facets having a size of 

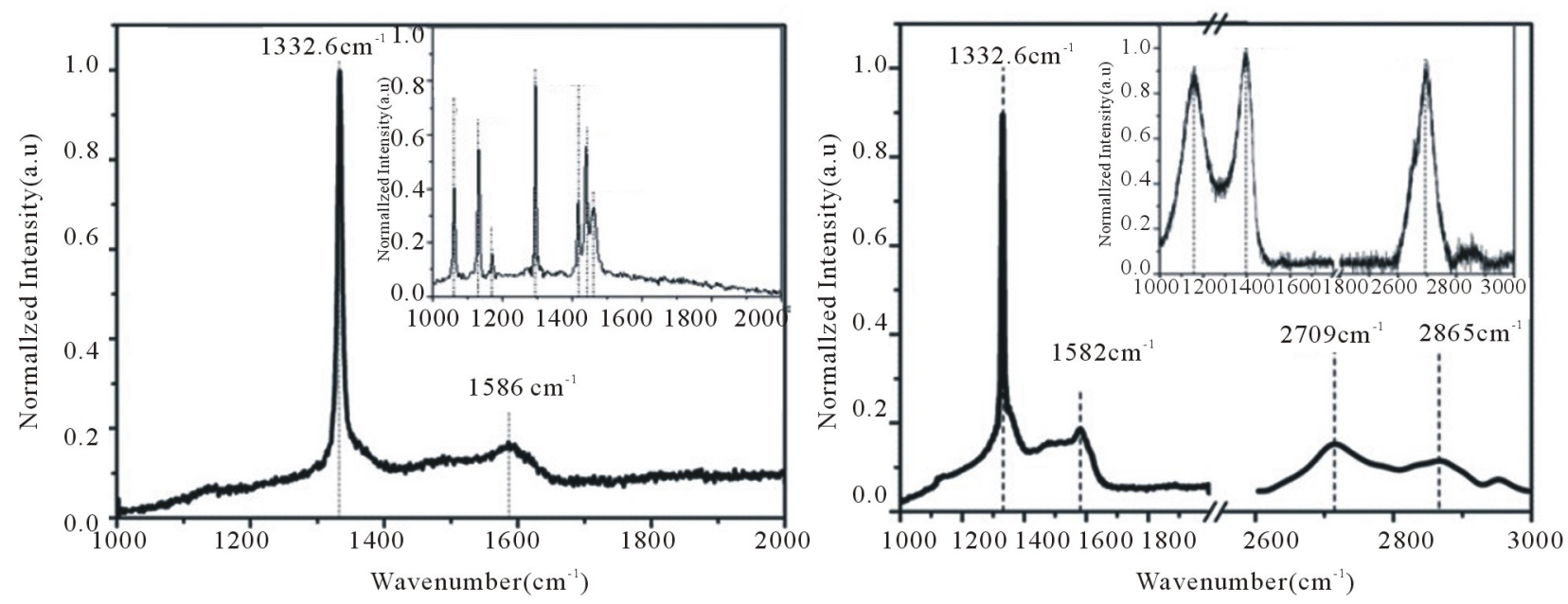

Figure 1. Raman spectra of (a) diamond film and (b) diamond-graphene film. Inset of Figure 1(a) shows the Raman spectrum of paraffin wax. Inset of Figure 1(b) shows the Raman spectrum of pre-synthesized graphene.

approximately $0.5-1.5 \mu \mathrm{m}$. Figure 2(b) is a SEM image of the pre-synthesized graphene used as a precursor in the fabrication of the hybrid film. Figure 2(c) shows a SEM image of the hybrid material revealing randomly nucleating diamond microcrystals (sizes ranging from 0.5 to $2.0 \mu \mathrm{m}$ ) embedded onto the graphene flakes. The small particles residing on the matrix material are the sub micrometer diamond crystallites resulting from the seeding source (paraffin wax) that are in the intermediate stage of diamond growth.

\subsection{Transmission Electron Microscopy}

The diamond and graphene-diamond hybrid films were further analyzed using TEM. Figure 3(a) shows a TEM image of the diamond film synthesized using paraffin wax as a seeding source. The corresponding selected area electron diffraction (SAED) pattern (inset of Figure 3(a)) confirms the structure of diamond with its $\{111\},\{220\}$ and $\{311\}$ reflections indexed (approximately $2.06 \AA$, $1.26 \AA$ and $1.08 \AA ̊$, respectively). Figure 3(b) shows a TEM image recorded at $30 \mathrm{kV}$, of the pre-synthesized graphene freely suspended on grid. The graphene sheets are thin and typically contain wrinkles and rolled edges. Figure 3(c) shows a TEM image of the hybrid film, indicating the presence of layered graphene together with the diamond microcrystals adhered to the graphene surface. In order to clearly establish the nature of carbon material grown on the copper substrate, electron energy loss spectroscopy (EELS) spectra were acquired and are shown in Figure 4. The spectra were recorded on different regions of graphene-diamond hybrid films comprising of diamond crystals, graphene flakes and also the pure amorphous carbon support shown in the inset of Figure 4.

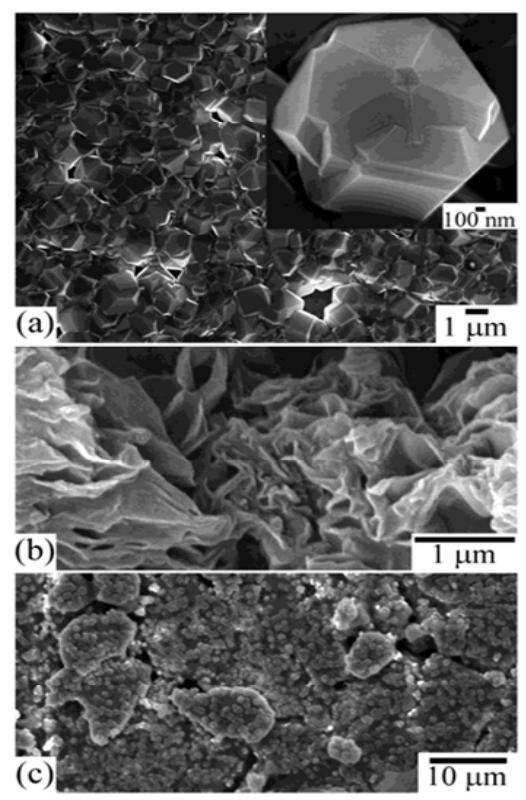

Figure 2. (a) Overview SEM image of the microcrystalline diamond film. The inset shows a diamond crystal with well-defined facets; (b) SEM image of the pre-synthesized graphene used as a precursor in the fabrication of the diamond-graphene hybrid material; (c) SEM image of the diamond-graphene hybrid material. The diamond microcrystals randomly nucleate onto the graphene flakes.

\subsection{Electron Energy Loss Spectroscopy}

EELS can be used to unambiguously distinguish between the different carbon materials [21] and was used to detect the elemental composition of the hybrid films. All the allotropes of carbon generally show K-absorption near edge structure between $280 \mathrm{eV}-320 \mathrm{eV}$. The EELS spectrum for the denser regions (as shown in inset of Figure 4 (blue line)) shows the main band around $292 \mathrm{eV}$ due to 


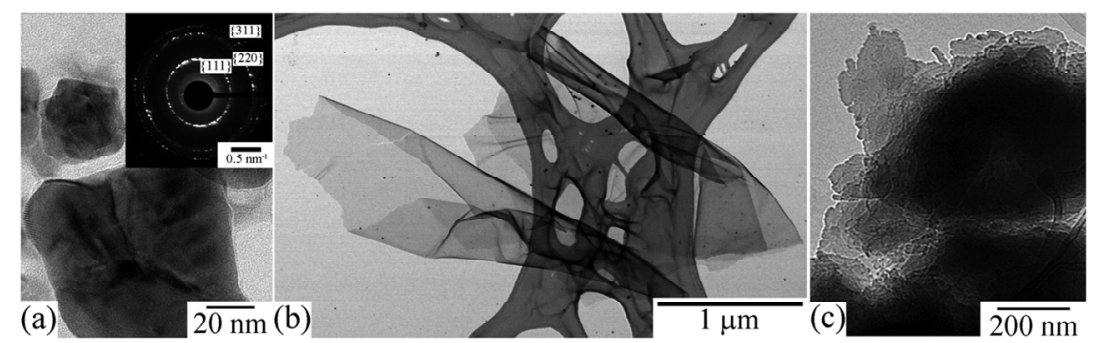

Figure 3. (a) TEM image showing the diamond crystals obtained from the diamond film synthesized using paraffin wax as a seeding source. The inset shows the corresponding electron diffraction pattern indexed to diamond with its $\{111\}$, $\{220\}$ and $\{311\}$ reflections. (b) TEM image of the pre-synthesized graphene freely suspended on grid (note the carbon support belonging to the grid). (c) TEM image of the hybrid material.

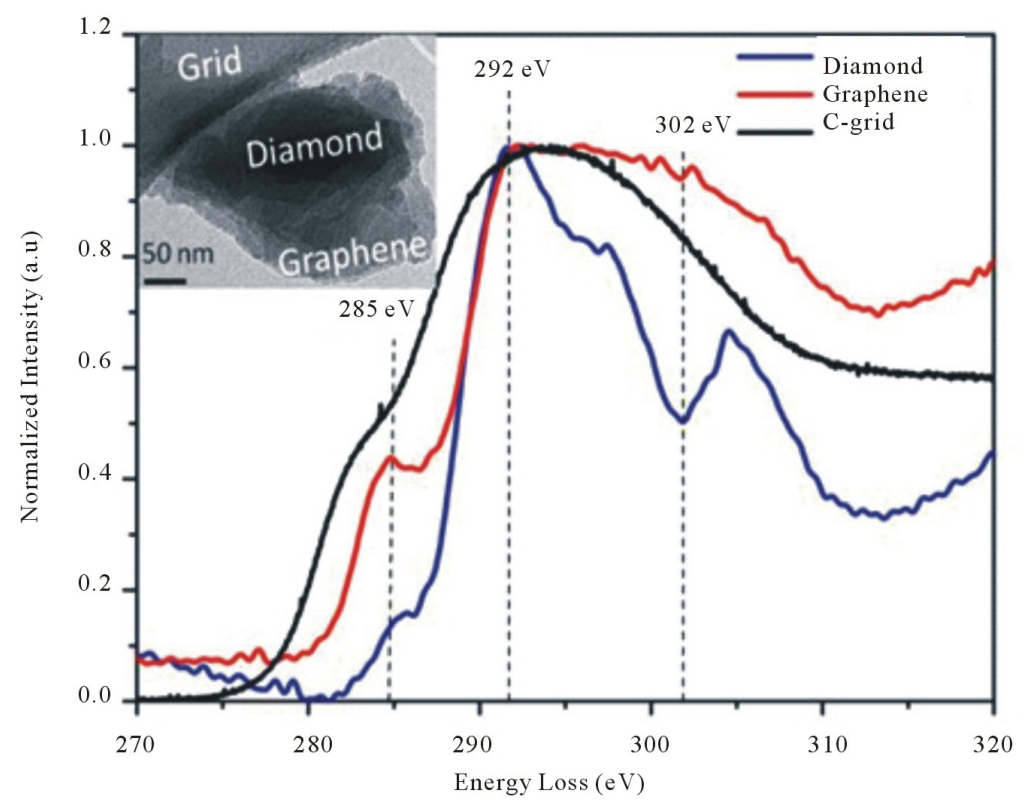

Figure 4. Electron energy loss spectrum on the region shown in the inset (red line for graphene, blue line for diamond and black line for carbon grid).

the $1 \mathrm{~s}-\sigma^{*}$ transition [33] and a dip around $302 \mathrm{eV}$, which is a characteristic feature of crystalline diamond [34]. The small shoulder ca. $285 \mathrm{eV}$ in the diamond spectrum may be arising due to the neighboring graphene flakes or due to the presence of $\mathrm{sp}^{2}$ graphitic carbon at the grain boundaries [35]. The EELS spectra of the semi-transparent zones (red line) exhibits the $1 \mathrm{~s}-\pi^{*}$ and $1 \mathrm{~s}-\sigma^{*}$ peaks at 285 and $292 \mathrm{eV}$ confirming the presence of graphene layers. In order to differentiate the obtained graphene spectrum from a-C, we took the EELS of the lacey carbon grid (a-C) shown in Figure 4 (black line) revealing a broad and structure less peak, owing to the fact that the local six fold symmetry is lost in the amorphous state [36]. Thus, the fabrication of the present hybrid material is evident from the TEM and EELS analyses.

\subsection{Electron Field Emission}

\subsubsection{J-E Curve}

The plot of the measured current density as a function of the macroscopic electrical field is shown in Figure 5. The field emission properties of the fabricated diamond films were reported previously by our group $[18,20]$. The field emission measurements reveal a threshold field of $3.6 \mathrm{~V} / \mu \mathrm{m}$ with a current density approaching $1 \mathrm{~mA} / \mathrm{cm}^{2}$ at $17-18 \mathrm{~V} / \mu \mathrm{m}$ for graphene-diamond hybrid film (Figure 5). A low threshold is observed due to the high geometrical factor of graphene flakes that cause local field enhancement and availability of conducting electrons in graphene, which is absent in case of diamond at room temperature. The plateau in emission current occurs at about $18 \mathrm{~V} / \mu \mathrm{m}$ for a current of about $10^{-5} \mathrm{~A}(0.01 \mathrm{~mA})$.

\subsubsection{F-N Plot}

The results of the electron emission studies can be analyzed in terms of the Fowler-Nordheim (F-N) theory [37]. In Figure 6, the F-N plot shows a bend in the downward direction that is often a feature of the carbon-based materials which can be attributed to the quantum tunneling of 
electrons through multiple barriers caused by the presence of different materials $[38,39]$. The Fowler-Nordheim plot for graphene-diamond hybrid has two slopes representing electron emission from two different regions. In region $20<\mathrm{E}<4.5 \mathrm{~V} / \mu \mathrm{m}$ the major contribution is due to electrons that are present in the conduction minima of graphene-diamond hybrid film and in the other, $4.5<\mathrm{E}<2.7 \mathrm{~V} / \mu \mathrm{m}$, the major contribution is made by the low occupancy states. An effective field enhancement factor $(\beta)$ calculated from the slopes of FN plot for

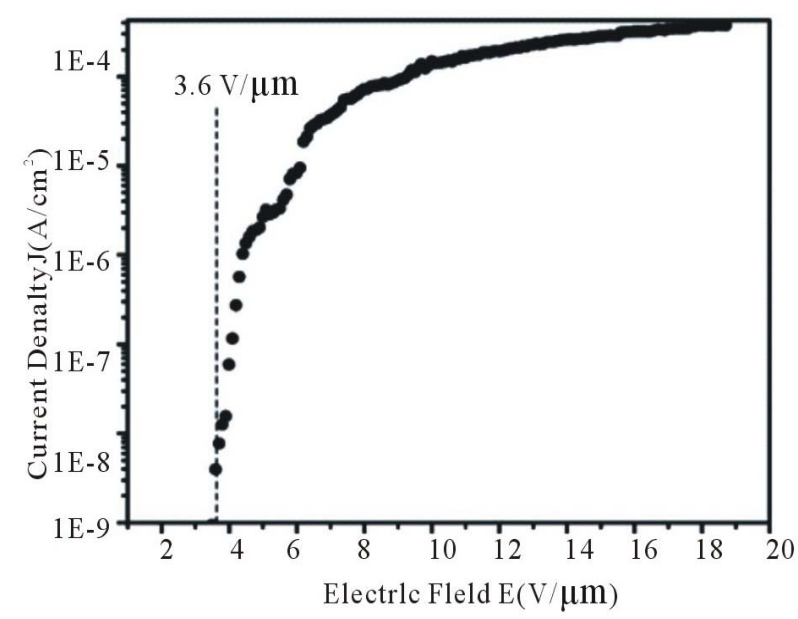

Figure 5. Plot of the measured field emission current as a function of the electric field for the graphene-diamond hybrid films. graphene-diamond hybrid (Figure 6) is calculated to be $7.05 \times 10^{2}$ and $2.99 \times 10^{3}$ for the two slopes. An enhancement of the field for hybrid films is brought about by dielectric inhomogeneities originating from the differences between conductive, spatially localized $\mathrm{sp}^{2} \mathrm{C}$ clusters surrounded by a more insulating $\mathrm{sp}^{3} \mathrm{C}$ matrix [40].

\subsubsection{Current Stability Test}

To evaluate the stability of the emission from the hybrid film, the current was monitored over a period of $135 \mathrm{~h} \mathrm{(7}$ days) with an initial current of the order of $0.1 \mathrm{~mA}$ at a field of $15 \mathrm{~V} / \mu \mathrm{m}$. The result obtained is shown in Figure 7. Superior emission stability was observed that can be explained on the basis of theoretical models proposed for emission from materials having both $\mathrm{sp}^{2}$ and $\mathrm{sp}^{3}$ hybridized carbon [41]. These models highlight the complimentary role of graphite-like $\left(\mathrm{sp}^{2}\right)$ region as emission center that is heated by the ohmic current passing through it and diamond-like $\left(\mathrm{sp}^{3}\right)$ region as heat sink that removes heat from the emission center. Although graphene has very high thermal conductivity, in the absence of better heat sink, most of the heat gets dumped at the graphene/substrate interface leading to the destruction of emission sites. However, in the presence of diamond, this excess heat gets removed very efficiently maintaining the integrity of the emission sites. The net result is a robust cold cathode material.

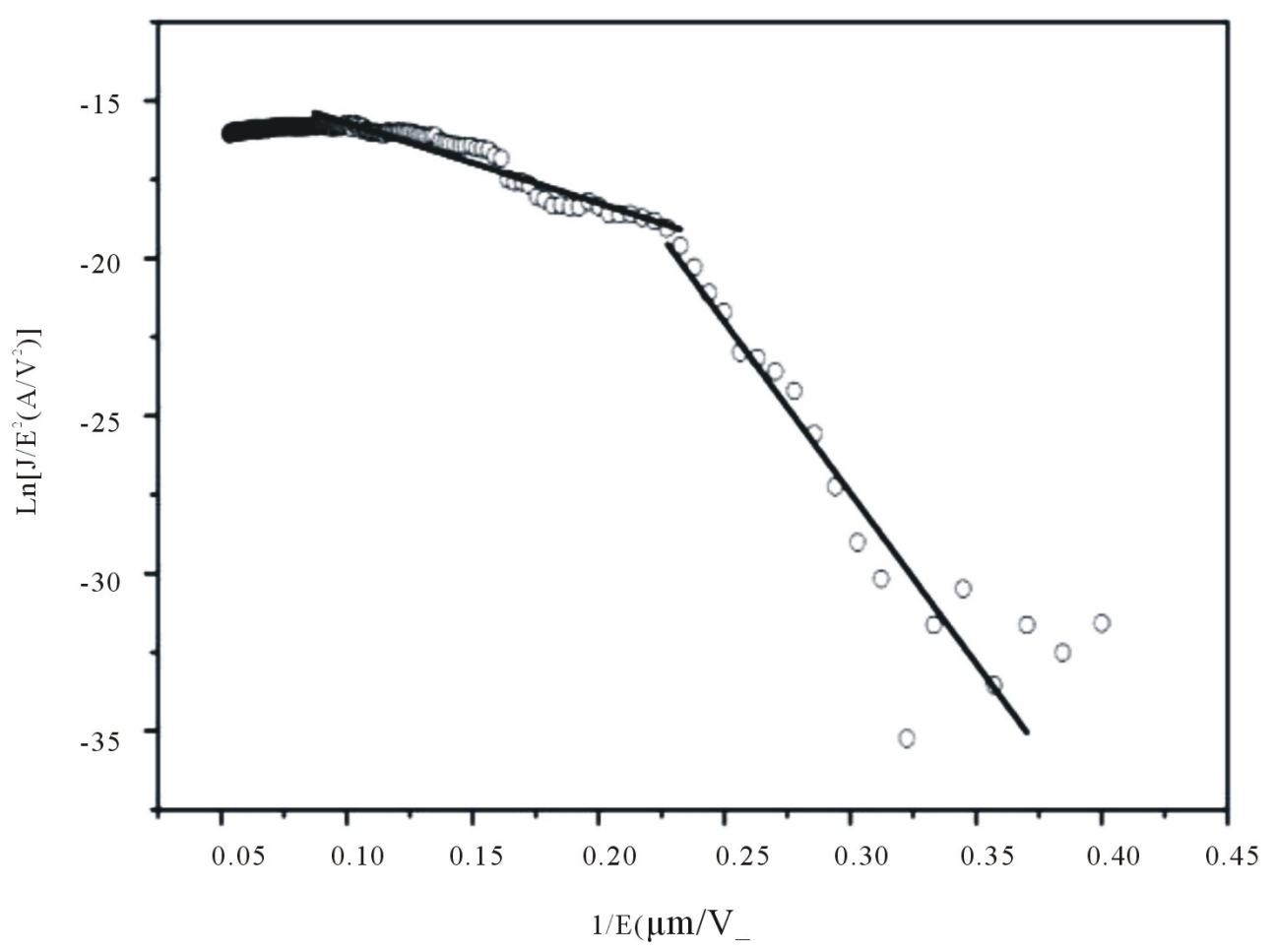

Figure 6. Fowler-Nordheim $\left(\ln \left(I / V^{2}\right)\right.$ vs $\left.V^{-1}\right)$ plot for the graphene-diamond hybrid films. 


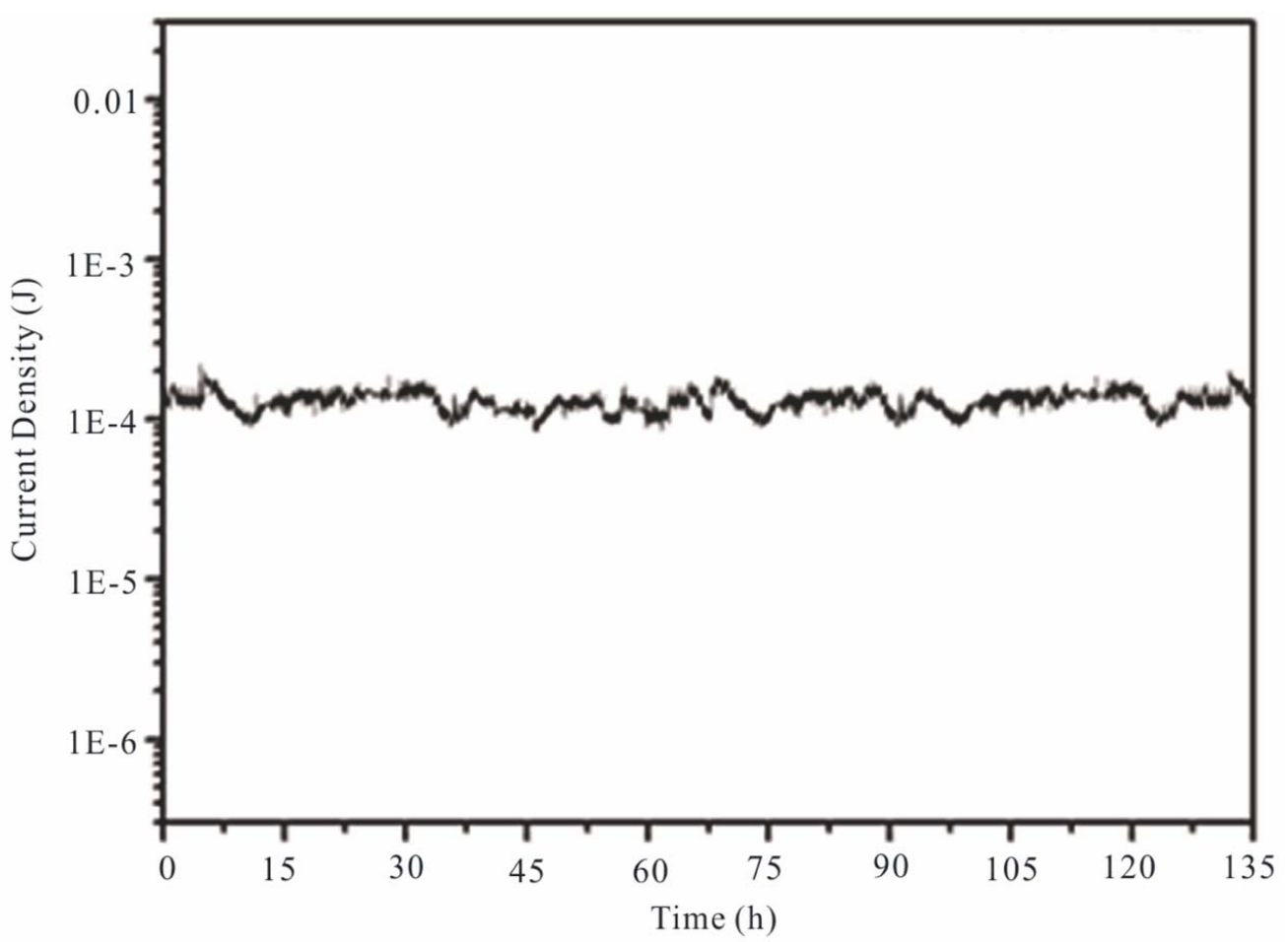

Figure 7. Plot of the emission current density versus time for a period of $135 \mathrm{~h}$ recorded from the graphene-diamond hybrid film.

The diamond-graphene hybrid film of this study shows better emission stability as compared to that of pure graphene films or graphene composite thin films (Table 1). In order to confirm the reproducibility of field emission characteristics, I-V measurements were performed at several locations. The observed field emission characteristics were almost independent of the locations, due to nearly uniform fabrication over the whole substrate surface.

\section{Conclusion}

Diamond and graphene-diamond hybrid films were fabricated by the HFCVD technique using paraffin wax as a seeding material for diamond. The characteristic bands of diamond and graphene were evident from Raman spectroscopy. Microscopic images reveal the presence of mi- cron-sized diamond crystals in the fabricated films. The graphene-diamond hybrid films exhibits a turn-on field of $3.8 \mathrm{~V} / \mu \mathrm{m}$ with an emission current density approaching $0.3 \mathrm{~mA} / \mathrm{cm}^{2}$ at a field of $20 \mathrm{~V} / \mu \mathrm{m}$. The hybrid film exhibits good emission current stability of $135 \mathrm{~h}$. The present study provides an inexpensive fabrication approach towards a graphene-diamond hybrid films that exhibit excellent field emission properties and can be a competitive candidate for efficient field emitter material.

\section{Acknowledgements}

This research was made possible by funds from the Institute for Functional Nanomaterials (NSF Grant \# 1002410), PR NASA EPSCoR (NASA Cooperative Agreement \# NNX07AO30A and NNX08BA48A), and

Table 1. Comparison of emission characteristics of present graphene-diamond hybrid film with existing literature.

\begin{tabular}{|c|c|c|c|c|}
\hline & $\begin{array}{l}\text { Field ehan cment } \\
\text { factor }\end{array}$ & $\begin{array}{l}\text { Turn-on } \\
\text { Field } \\
(\mathrm{V} / \mu \mathrm{m})\end{array}$ & $\begin{array}{l}\text { Current stability } \\
\text { (minutes) }\end{array}$ & Reference \\
\hline Graphene paper & $3.2 \times 10^{3}-4.1 \times 10^{3}$ & $1.6-2.4$ & $1500-1700$ & {$[42]$} \\
\hline $\begin{array}{l}\text { Graphene on } \\
\text { polymer film }\end{array}$ & $1.0 \times 10^{3}$ & 1.8 & $>180$ & {$[43]$} \\
\hline $\begin{array}{c}\text { Graphene-diamond } \\
\text { Hybrid material }\end{array}$ & $5.9 \times 10^{2}-1.1 \times 10^{4}$ & 3.8 & $>8100$ & Present work \\
\hline
\end{tabular}


PR DOE EPSCoR (DOE Grant \# DEFG02-08ER46526). D.V would like to acknowledge the help of Mr. W. Pérez for the Raman spectroscopy measurements. Microscopes are operated at the Nanoscopy facility at UPR.

\section{REFERENCES}

[1] K. Baba, Y. Aikawa, N. Shohata, H. Yoneda and K. I. Ueda, "Photo Conductive Switch with CVD Diamond Films by Ultraviolet Light Pulse," NEC Research Development, Vol. 36, No. 3, 1995, pp. 369-375. doi:10.1016/0925-9635(95)00448-3

[2] L. S. Pan and D. R. Kania, "Diamond: Electronic Properties and Applications: Kluwer Academic Publishers," Kluwer Academic Publishers, Boston, 1995.

[3] D. A. David, R. Epstein and R. Hanson, "The Diamond Age of Spintronics," Scientific American, Vol. 297, No. 4, 2007, pp. 84-91. doi:10.1038/scientificamerican1007-84

[4] B. R. Stoner, G. H. M. Ma, S. D. Wolter and J. T. Glass, "Characterization of Bias-Enhanced Nucleation of Diamond on Silicon by Invacuo Surface Analysis and Transmission Electron Microscopy," Physical Review B, Vol. 45, No. 19, 1992, pp. 11067-11084. doi:10.1103/PhysRevB.45.11067

[5] K. Uppireddi, O. Resto, B. R. Weiner and G. Morell, "Iron Oxide Nanoparticles Employed as Seeds for the Induction of Microcrystalline Diamond Synthesis," $\mathrm{Na}$ noscale Research Letters, Vol. 3, No. 2, 2008, pp. 65-70. doi:10.1007/s11671-008-9117-5

[6] S. Biwu and W. M. L. Leo, "Apparatus and Method for Nucleation and Deposition of Diamond Using Hot-Filament Dc Plasma,” US Patent No. 6161499, 2000.

[7] G. Alfred, L. C. Robert and J. D. Patrick, "Grown Diamond Mosaic Separation," US Patent No. 2007/0017437, 2007.

[8] Q. Wang, R. Schliesing, H. Zacharias and V. Buck, "Enhancement of Diamond Nucleation on Silicon Substrates in Pulsed Laser Assisted Hot Filament CVD," Applied Surface Science, Vol. 138-139, 1999, pp. 429-433. doi:10.1016/S0169-4332(98)00436-X

[9] K. S. Novoselov, A. K. Geim, S. V. Morozov, D. Jiang, Y. Zhang, S. V. Dubonos, I. V. Grigorieva and A. A. Firsov, "Electric Field Effect in Atomically Thin Carbon Films," Science, Vol. 306, No. 5696, 2004, pp. 666-669. doi:10.1126/science. 1102896

[10] X. Wang, L. J. Zhi and K. Mullen, "Transparent, Conductive Graphene Electrodes for Dye-Sensitized Solar Cells," Nano Letters, Vol. 8, No. 1, 2008, pp. 323-327. doi:10.1021/n1072838r

[11] G. Eda and M. Chhowalla, "Graphene-Based Composite Thin Films for Electronics," Nano Letters, Vol. 9, No. 2, 2009, pp. 814-818. doi:10.1021/n18035367

[12] K. Okano, S. Koizumi, S. R. P. Silva and G. A. J. Amaratunga, "Low-Threshold Cold Cathodes Made of Nitrogen-Doped Chemical-Vapour-Deposited Diamond," $\mathrm{Na}$ ture, Vol. 381, No. 9, 1996, pp. 140-141. doi: $10.1038 / 381140 \mathrm{a} 0$
[13] G. A. J. Amaratunga and S. R. P. Silva, "Nitrogen Containing Hydrogenated Amorphous Carbon for Thin-Film Field Emission Cathodes," Applied Physics Letters, Vol. 68 , No. 18, 1996, pp. 2529-2531. doi: $10.1063 / 1.116173$

[14] W. A. De Heer, A. Chatelain and D. Ugarte, "A Carbon Nanotube Field-Emission Electron Source," Science, Vol. 270, No. 5239, 1995, pp. 179-180. doi:10.1126/science.270.5239.1179

[15] R. B. Rakhi, A. L. M. Reddy, M. M. Shaijumon, K. Sethupati and S. Ramaprabhu, "Electron Field Emitters Based on Multi-Walled Carbon Nanotubes Decorated with Nanoscale Metal Clusters," Journal of Nanoparticle Research, Vol. 10, No. 1, 2008, pp. 179-189. doi:10.1007/s11051-007-9240-8

[16] G. Eda, H. E. Unalan, N. Rupesinghe, G. A. J. Amaratunga and M. Chhowalla, "Field Emission from Graphene Based Composite Thin Films," Applied Physics Letters, Vol. 93, No. 23, 2008, pp. 233502-233505. doi:10.1063/1.3028339

[17] A. Malesevic, R. Kemps, A. Vanhulsel, M. P. Chowdhury, A. Volodin and C. V. Haesendonck, "Field Emission from Vertically Aligned Few-Layer Graphene," Journal of Applied Physics, Vol. 104, No. 8, 2008, pp. 084301084305. doi:10.1063/1.2999636

[18] D. Varshney, V. I. Makarov, P. Saxena, M. J.-F. Guinel, A. Kumar, J. F. Scott, B. R. Weiner and G. Morell, "Electron Emission from Diamond Films Seeded Using Kitchen-Wrap Polyethylene," Journal of Physics D: Applied Physics, Vol. 44, No. 8, 2010, pp. 085502-085508. doi:10.1088/0022-3727/44/8/085502

[19] K. Uppireddi, C. V. Rao, Y. Ishikawa, B. R. Weiner and G. Morell, "Temporal Field Emission Current Stability and Fluctuations from Graphene Films," Applied Physics Letters, Vol. 97, No. 6, 2010, pp. 62106-62108. doi: $10.1063 / 1.3474800$

[20] G. Morell, A. Gonzalez-Berrios, B. R. Weiner and S. Gupta, "Synthesis, Structure, and Field Emission Properties of Sulfur-Doped Nanocrystalline Diamond," Journal of Materials Science: Materials in Electronics, Vol. 17, No. 6, 2006, pp. 443-451.

[21] A. González-Berríos, F. Piazza and G. Morell, "Effects of Adsorbates on Field Emission Reproducibility of Sulfur-Incorporated Nanocomposite Carbon Films," Journal of Vacuum Science \& Technology B, Vol. 25, No. 2, 2005, pp. 318-323.

[22] Y. Wang, M. Rafailovich, J. Sokolov, D. Gersappe, T. Araki, Y. Zou, A. D. L. Kilcoyne, H. Ade, G. Marom and A. Lustiger, "Substrate Effect on the Melting Temperature of Thin Polyethylene Films," Physical Review Letters, Vol. 96, No. 2, 2006, pp. 028303-028306. doi:10.1103/PhysRevLett.96.028303

[23] Z. Stephen and D. Cheng, "Materials Science: Polymer Crystals Downsized," Nature, Vol. 448, No. 157, 2007, pp. 1006-1007. doi:10.1038/4481006a

[24] M. Zheng and W. Du, "Phase Behavior, Conformations, Thermodynamic Properties, and Molecular Motion of Multicomponent Paraffin Waxes: A Raman Spectroscopy 
Study," Vibrational Spectroscopy, Vol. 40, No. 2, 2006, pp. 219-224.

[25] J. E. Field, "The Properties of Diamond," Academic Press, London, 1979, pp. 281-324.

[26] D. Varshney, C. V. Rao, M. J.-F. Guinel, Y. Ishikawa, B. R. Weiner and G. Morell, "Free Standing GrapheneDiamond Hybrid Films and Their Electron Emission Properties," Journal of Applied Physics, Vol. 110, No. 4, 2011, pp. 044324-0443249. doi:10.1063/1.3627370

[27] M. A. Pimenta, G. Dresselhaus, M. S. Dresselhaus, L. G. Cancado, A. Jorio and R. Saito, "Studying Disorder in Graphite-Based Systems by Raman Spectroscopy," Physical Chemistry Chemical Physics, Vol. 9, No. 11, 2007, pp. 1276-1290. doi:10.1039/b613962k

[28] A. C. Ferrari, J. C. Meyer, V. Scardaci, C. Casiraghi, M. Lazzeri, F. Mauri, S. Piscanec, D. Jiang, K. S. Novoselov, S. Roth and A. K. Geim, "Raman Spectrum of Graphene and Graphene Layers," Physical Review Letters, Vol. 97, No. 18, 2006, pp. 187401-187403. doi:10.1103/PhysRevLett.97.187401

[29] C. Thomsen and S. Reich, "Double Resonant Raman Scattering in Graphite," Physical Review Letters, Vol. 85, No. 25, 2000, pp. 5214-5217.

[30] L. M. Malard, M. A. Pimenta, G. Dresselhaus and M. S. Dresselhaus, "Raman Spectroscopy in Grapheme," Physics Reports, Vol. 473, No. 5-6, 2009, pp. 51-87. doi:10.1016/j.physrep.2009.02.003

[31] J. Wang, M. Zhu, R. A. Outlaw, X. Zhao, D. M. Manos and B. C. Holloway, "Synthesis of Carbon Nanosheets by Inductively Coupled Radio-Frequency Plasma Enhanced Chemical Vapor Deposition," Carbon, Vol. 42, No. 14, 2004, pp. 2867-2872. doi:10.1016/j.carbon.2004.06.035

[32] A. Reina, X. Jia, J. Ho, D. Nezich, H. Son, V. Bulovic, M. S. Dresselhaus and J. Kong, "Large Area, Few-Layer Graphene Films on Arbitrary Substrates by Chemical Vapor Deposition," Nano Letters, Vol. 9, No. 1, 2009, pp. 30-35. doi: $10.1021 / \mathrm{nl} 801827 \mathrm{~V}$

[33] D. Varshney, B. R. Weiner and G. Morell, "Growth and Field Emission Study of a Monolithic Carbon Nanotube/Diamond Composite," Carbon, Vol. 48, No. 18, 2010, pp. 3353-3358. doi:10.1016/j.carbon.2010.05.025

[34] R. Arenal, P. Bruno, D. J. Miller, M. Bleuel, J. Lal and D. M. Gruen, "Diamond Nanowires and the Insulator-Metal Transition in ultrananocrystalline Diamond Films," Phy- sical Review B, Vol. 75, No. 19, 2007, pp. 195431195441. doi:10.1103/PhysRevB.75.195431

[35] J. E. Butler and A. V. Sumant, "The CVD of Nanodiamond Materials," Chemical Vapor Deposition, Vol. 14, No. 7-8, 2008, pp. 145-160. doi:10.1002/cvde. 200700037

[36] S. Muto, T. Tanabe, A. Hirota, M. Rubel, V. Philipp and T. Maruyama, "TEM and EELS Characterization of Carbon Dust and Co-Deposited Layers from the TEXTOR Tokamak," Journal of Nuclear Materials, Vol. 307-311, 2002, pp. 1289-1293. doi:10.1016/S0022-3115(02)01118-2

[37] R. H. Fowler and L. Nordheim, "Electron Emission in Intense Electric Fields," Proceedings of the Royal Society of London. Series A, Vol. 119, No. 781, 1928, pp. 173181.

[38] C. Ducati, E. Barborini, P. Piseri, P. Milani and J. Robertson, "Influence of Cluster-Assembly Parameters on the Field Emission Properties of Nanostructured Carbon Films," Journal of Applied Physics, Vol. 92, No. 9, 2002, pp. 5482-5489. doi:10.1063/1.1512969

[39] M. Liao, Z. Zhang, W. Wang and K. Liao, "Field-Emission Current from Diamond Film Deposited on Molybdenum," Journal of Applied Physics, Vol. 84, No. 2, 1998, pp. 1081-1084. doi:10.1063/1.368096

[40] J. D. Carey, R. D. Forrest and S. R. P. Silva, "Origin of Electric Field Enhancement in Field Emission from Amorphous Carbon Thin Films," Applied Physics Letters, Vol. 78, No. 16, 2001, pp. 2339-2341. doi:10.1063/1.1366369

[41] E. D. Eidelman and A. Y. Vul, "The Strong Thermoelectric Effect in Nanocarbon Generated by the Ballistic Phonon Drag of Electrons," Journal of Physics: Condensed Matter, Vol. 19, No. 26, 2007, pp. 266210-266221.

[42] J. Liu, B. Zeng, Z. Wu, J. Zhu and X. Liu, "Improved Field Emission Property of Graphene Paper by Plasma Treatment," Applied Physics Letters, Vol. 97, No. 3, 2010, pp. 033109-033111. doi:10.1063/1.3467042

[43] V. P. Verma, S. Das, I. Lahiri and W. Choi, "Large-Area Graphene on Polymer Film for Flexible and Transparent Anode in Field Emission Device," Applied Physics Letters, Vol. 96, No. 20, 2010, pp. 203108-203110. 